\title{
The Effect of Phase Decomposition on Magnetic Structure of $\mathrm{Cu}_{0.4} \mathrm{Mn}_{0.3} \mathrm{Ni}_{0.3}$ Alloy
}

\author{
K. ŚWIDERSKA* AND J. JANKOWSKA-KISIELIŃSKA \\ National Centre for Nuclear Research, 05-400 Otwock-Świerk, Poland
}

\begin{abstract}
The purpose of present investigation was to study the effects of phase decomposition of the $\mathrm{Cu}_{0.4} \mathrm{Mn}_{0.3} \mathrm{Ni}_{0.3}$ alloy on its magnetic ordering. The single-crystal sample was examined by elastic neutron scattering before and after five subsequent annealing runs. The results indicate that in small fraction of the quenched sample volume there are two types of antiferromagnetic order: one of them AF1 of the long range, the other one of the short range. Ageing induces phase decomposition which yields disappearance of AF1 long range order and increase of the volume of new ordered phase. The results of the investigations of the aged sample indicate that regions of new tetragonal phase exhibit antiferromagnetic ordering with magnetic moments of $\mathrm{Mn}$ atoms arranged as in the pure compound $\mathrm{MnNi}$ but with unequal domain distribution. The intensity of magnetic component of super-structure reflections increases with the duration of ageing at lower rate than component due to atomic order.
\end{abstract}

DOI: 10.12693/APhysPolA.127.394

PACS: 61.05.F-, 64.75.-g, 75.40.-s, 75.47.Np, 75.50.Ee, 81.40.Rs

\section{Introduction}

Pseudo quasi-binary $\mathrm{Cu}_{1-2 x} \mathrm{Mn}_{x} \mathrm{Ni}_{x}$ alloys are among those known to harden in effect of decomposition which results in atomic and magnetic ordering and tetragonal distortion in one of the phases [1-3]. The early investigation of these alloys [1] indicated that decomposition is spinodal. However more detailed studies [2,3] revealed random distribution of new phase precipitates at the beginning stage of decomposition indicating the nucleation rather than spinodal mode of decomposition.

The atomically disordered $\mathrm{Cu}_{1-2 x} \mathrm{Mn}_{x} \mathrm{Ni}_{x}$ alloy of the fcc structure can be achieved by annealing at 1070$1220 \mathrm{~K}$ and a subsequent quenching [1, 3]. Annealing at $670-770 \mathrm{~K}$ induces the decomposition into two phases [1-3]. The new phase, of a lowered symmetry tetragonal structure, is poor in $\mathrm{Cu}$ atoms and rich in atomically ordered intermetallic compound $\mathrm{MnNi}$. The coexistent phase of the fcc structure is a solid solution, rich in $\mathrm{Cu}$ atoms and poor in $\mathrm{Mn}$ and $\mathrm{Ni}$.

The aim of present work was to determine the changes in magnetic ordering of the $\mathrm{Cu}_{0.4} \mathrm{Mn}_{0.3} \mathrm{Ni}_{0.3}$ alloy at the beginning stage of decomposition. The investigation was conducted for the quenched sample and after subsequent ageing runs. The expected atomic and magnetic structure of the ordered phase was that of the $\mathrm{MnNi}$ compound [1-4]. The atomic structure of the MnNi compound is known to be tetragonal $P 4 / \mathrm{mmm}$, and magnetic structure to be orthorhombic $P_{C}$ man or $C_{a} m m a$. $P_{C}$ man structure contains 4 atoms in unit cell: $\mathrm{Mn}$ atom with spin in the [100] direction at $(0,0,0)$, Mn atom with spin in the $[-100]$ direction at $(1 / 2,1 / 2,0)$ and $\mathrm{Ni}$ atoms with practically no magnetic moments at $(1 / 2,0,1 / 2)$

\footnotetext{
* corresponding author; e-mail: karolina.swiderska@ncbj.gov.pl
}

and $(0,1 / 2,1 / 2,) . C_{a} m m a$ structure contains 8 atoms in unit cell: $\mathrm{Mn}$ atoms with spin in [100] direction at $(0,0,0)$ and $(1 / 2,1 / 2,0), \mathrm{Mn}$ atoms with spin in the [100 direction at $(1 / 2,0,0)$ and $(0,1 / 2,0)$, $\mathrm{Ni}$ atoms with practically no magnetic moments at $(1 / 4,1 / 4,1 / 2)$, $(1 / 4,3 / 4,1 / 2),(3 / 4,1 / 4,1 / 2)$, and $(3 / 4,3 / 4,1 / 2)[5]$.

\section{Sample and experimental method}

The sample was a single-crystal of $\mathrm{Mn}, \mathrm{Ni}$ and $\mathrm{Cu}$ (30 at.\% Mn, 30 at.\% Ni, 40 at.\% Cu) alloy in the form of a cube with dimensions $1 \mathrm{~cm} \times 1 \mathrm{~cm} \times 1 \mathrm{~cm}$. In order to homogenize the sample it was annealed at $1170 \mathrm{~K}$ and afterwards quenched by immersing in cold water. The sample was then subjected to five subsequent runs of annealing at 700-730 K: $0.5 \mathrm{~h}, 0.5 \mathrm{~h}, 1 \mathrm{~h}, 2 \mathrm{~h}$, and $4 \mathrm{~h}$.

The reflections obtainable in the (100) and (110) scattering planes were initially investigated in the quenched sample by neutrons with $\lambda=1.5 \AA$. Similar measurements were repeated after the last annealing. More detailed measurements at (100), (110), (210) and (200) were performed for the neutron wavelengths of $2.35 \AA$ in the temperature range $15-290 \mathrm{~K}$ before the first and after every annealing.

The triple axis spectrometer (TAS) at the elastic mode was used. The $\lambda / 2$ contamination of the measured intensities, estimated from profile measured on $\mathrm{Al}_{2} \mathrm{O}_{3}$ standard sample, was $I(\lambda / 2) / I(\lambda)=0.00095 \pm 0.00015$ for $\lambda=2.35 \AA$ and $I(\lambda / 2) / I(\lambda)=0.0023 \pm 0.0003$ for $\lambda=1.5 \AA$.

\section{Results and data analysis}

The expected fcc structure of the quenched alloy was verified by elastic scattering of neutrons with the wavelength $\lambda=1.5 \AA$. The maximum intensities of the observed reflections are presented in Fig. 1a. The Bragg reflections expected for the fcc structure were found. 
A lattice constant is $a=3.659 \pm 0.001 \AA(T=$ $295 \mathrm{~K})$. In addition, we observed super-structure reflections of different (close to Lorentzian) shape and intensity two orders of magnitude lower. More detailed investigation for neutrons with $\lambda=2.35 \AA$ of the intensity distributions at (100), (110) and (210) reflections revealed their complex nature. The (100) and (110) reflections can be described as a sum of two components: Gaussian and Lorentzian. For both quenched and aged sample the sum of Gaussian and Lorentzian was fitted to the data with the least square method. The key parameters obtained for both functions in the process of data fitting were the intensity maximum $A_{\mathrm{G}}$ and $A_{\mathrm{L}}$, the corresponding value of scattering vector lengths $\left|Q_{\mathrm{G}}\right|$ and $\left|Q_{\mathrm{L}}\right|$ and the width of a distribution $\mathrm{FWHM}_{\mathrm{G}}$ and $\mathrm{FWHM}_{\mathrm{L}}$.

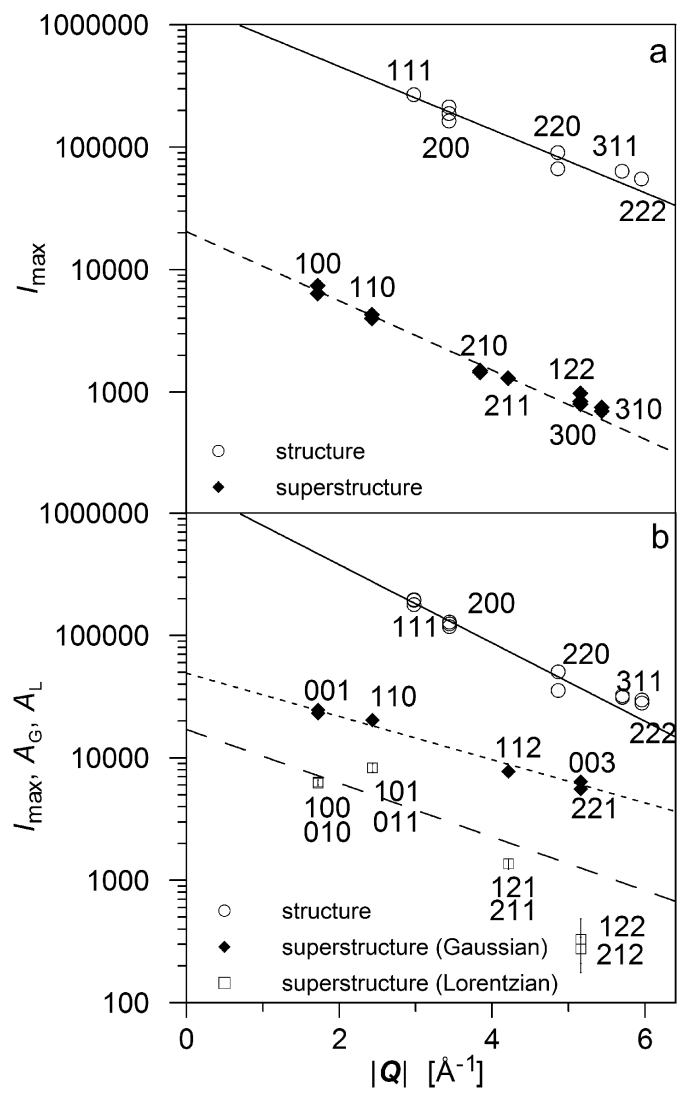

Fig. 1. (a) Intensity at maximum of the fcc Bragg reflections (open circles) and additional super-structure reflections (filled squares) versus scattering vector, measured at $295 \mathrm{~K}$ before sample annealing. Fits for structural and superstructure reflections: solid line $\log _{10}\left(I_{\max }\right)=6.2-0.26|Q|$, dashed line $-\log _{10}\left(I_{\max }\right)=$ $4.3-0.28|Q|$, respectively. (b) Intensity at maximum of the fcc Bragg reflections (open circles) and maximum of Gaussian (filled squares) and Lorentzian (open squares) components of super-structure reflections versus scattering vector, measured at $295 \mathrm{~K}$ after $8 \mathrm{~h}$ of sample annealing. Fits for fcc and superstructure (Gaussian and Lorentzian component) reflections: solid line $-\log _{10}\left(I_{\max }\right)=6.2-0.32|Q|$, dotted line $\log _{10}\left(I_{\max }\right)=4.7-0.21|Q|$, dashed line $-\log _{10}\left(I_{\max }\right)=$ $4.2-0.22|Q|$, respectively.
The measurements with neutrons of $\lambda=1.5 \AA$ performed after 5 sequential annealing runs of the total time $8 \mathrm{~h}$ confirmed the complexity of the superstructure reflections. Maximum intensities of the fcc reflections and of the components of superstructure reflections are shown in Fig. 1b for the sample after annealing.

Examples of the intensity distributions for (100) and (110) reflection measured with neutrons of $\lambda=$ $2.35 \AA$ at $15 \mathrm{~K}$ are shown in Fig. 2 for the sample after $4 \mathrm{~h}$ of annealing.

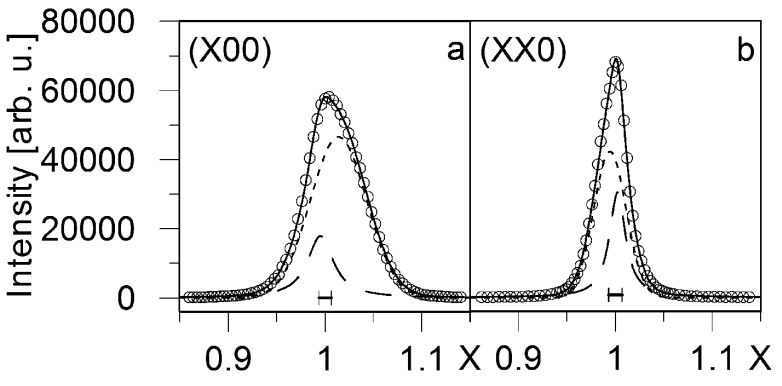

Fig. 2. The intensity distributions of neutrons scattered elastically on the $\mathrm{Cu}_{0.4} \mathrm{Mn}_{0.3} \mathrm{Ni}_{0.3}$ sample at 15 $\mathrm{K}$ for (100) reflection (a) and for (110) reflection (b), for the sample after $4 \mathrm{~h}$ of ageing . Circles - experimental points, solid line - fitted result, dotted line Gaussian component, dashed line - Lorentzian component. The spectrometer resolution is marked with a horizontal bar.

Temperature dependence of the intensity of the Gaussian and Lorentzian components of (100) and (110) reflections in the quenched sample is presented in Fig. 3.

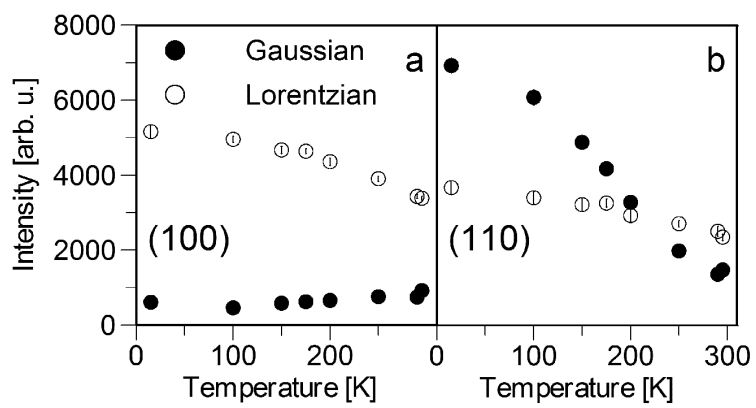

Fig. 3. The temperature dependence of the intensity of Gaussian and Lorentzian components of (100) reflection (a), and (110) reflection (b), for the quenched sample.

The very small and independent of temperature Gaussian component of (100) reflection is probably the contamination from (200) reflection for $\lambda / 2$.

The intensity of Gaussian component for the (110) reflection is large and strongly decreases with temperature. The character of the temperature dependence of the intensity of Gaussian component of the (110) reflection indicates its magnetic origin and ordering temperature 
below $300 \mathrm{~K}$. The width of this component of (110) reflection is close to the resolution of the spectrometer, proving the long correlation range of magnetic ordering. The absence of magnetic (100) reflection, and intensity relation of (110) and (201) indicate the AF1-type magnetism in some (small) fraction of the sample.

The intensity of Lorentzian component decreased a little with increasing temperature at both (100) and (110) reflections in similar way. The Lorentzian contribution was about four times wider than the resolution of the spectrometer suggesting short range ordering of another type in some small sample regions.

After annealing the Gaussian and Lorentzian components of superstructure reflections are placed at slightly different positions indicating tetragonal distortion of the structure of the new phase. In terms of the tetragonal structure the Gaussian components of first 5 superstructure reflections are (001), (110), (201), (112) and (221) and the Lorentzian components are (100), (011), (102), (211), and (122).

Lattice parameter of the main part of the sample obtained from the (200) reflection does not change. Tetragonal distortion of the new phase increases with ageing duration according to the expectations. At room temperature tetragonal distortion comes to the value $c / a=0.9800 \pm 0.0006$ after $8 \mathrm{~h}$ of annealing. In comparison, for intermetallic $\mathrm{MnNi}$ the reported tetragonal distortion is larger $-c / a=0.942$ [6]. The values of lattice parameters $a$ and $c$ obtained from (110) and (011) are consistent with that from (100) and (001) reflections. The positions of the Gaussian component of reflections (001), (110), (112) and (221) are consistent with position of atomic super-lattice reflections and the positions of Lorentzian component of reflections (100), (101), (211) and (122) are consistent with positions of magnetic reflections in pure $\mathrm{MnNi}$ compound [5]. We can assume that the origin of the Gaussian component is atomic ordering and the Lorentzian component is magnetic.

The intensity of Gaussian and Lorentzian components of the super-structure reflections increases with the total time of annealing, as shown in Fig. 4 for (100) and (110) reflections measured at $15 \mathrm{~K}$.

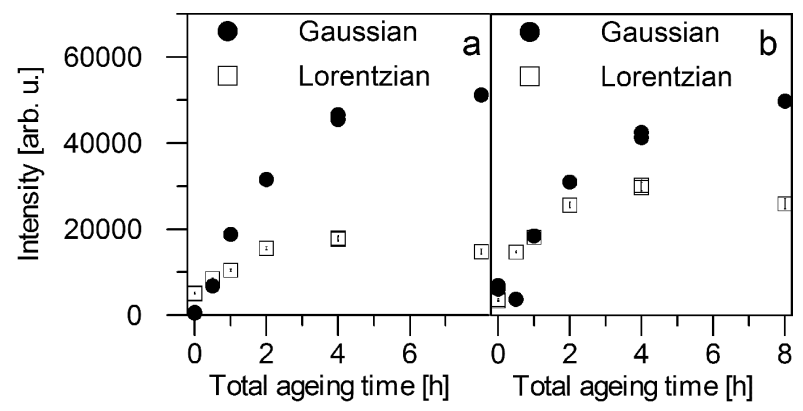

Fig. 4. Intensity of Gaussian and Lorentzian components of the super-structure reflections measured at $15 \mathrm{~K}$ : (a) for (100) and (b) for (110) reflections, versus time of sample annealing.
The intensity ratio $A_{\mathrm{L}}(100) / A_{\mathrm{L}}(101)$ of the first magnetic reflections is $\approx 0.6$ and $\approx 0.75$ for measurements after $1-4 \mathrm{~h}$ and $8 \mathrm{~h}$ of annealing, respectively. For the $P_{C}$ man and $C_{a} m m a$ structure of $\mathrm{MnNi}$ compound described in [5] this ratio equals 1 in the single-crystal sample with equal probability for different magnetic domains. The relation $A(100) / A(101)<1$ is not permitted for equal domain distribution for other magnetic structures considered in [5], however it is permitted for $P_{C}$ man structure with unequal domain distribution.

\section{Conclusions}

The investigations of the quenched sample have shown that quenching which aimed to prevent the initiation of the phase decomposition was probably insufficiently quick hence the quenched sample is not completely homogeneous. Two types of magnetic order are observed in very small parts of the sample volume. The first type is long range antiferromagnetic order, and the other one is short range antiferromagnetic order. After ageing the long range order AF1 disappears.

After ageing the long range antiferromagnetic order disappears. Different positions of Gaussian and Lorentzian components of the superstructure reflections of the new phase formed during the sample decomposition allowed (in analogy to intermetallic $\mathrm{MnNi}$ ) to assign the Gaussian - the atomic and to the Lorentzian the magnetic origin. The intensity ratio $A(100) / A(101)$ of the first magnetic reflections indicated the $P_{C}$ man structure with unequal probability of domain orientation. Preferable domain orientation may be connected with strains induced by tetragonal distortion of new phase precipitations in the fcc matrix. Inhomogeneous distribution of precipitations with 3 possible orientations of $c$ axis (1 or 2 preferable directions observed in different parts of single crystal samples) in $\mathrm{Cu}_{1-2 x} \mathrm{Mn}_{x} \mathrm{Ni}_{x}$ alloys [3] can induce additional preferential axis.

The intensities of all of the components increase with time of ageing suggesting the similar growth of the volume fraction of the ordered phase. However the intensity of the magnetic component decreases after first $0.5 \mathrm{~h}$ of ageing.

\section{References}

[1] J. Rolland, P. Priester, D. Whitwham, C.R. Acad. Sci. Paris C 270, 1777 (1970).

[2] D. Rondot, J. Mignot, Acta Metall. 26, 217 (1978).

[3] N.A. Polyakova, V.A. Udovenko, E.D. Chichua, Fiz. Metal. Metalloved. 71, 178 (1991) (in Russian).

[4] N.A. Polyakova, V.A. Udovenko, E.D. Chichua, Fiz. Metal. Metalloved. 66, 1144 (1988) (in Russian).

[5] J.S. Kasper, J.S. Kouvel, J. Phys. Chem. Solids 11, 231 (1959).

[6] E. Krén, E. Nagy, I. Nagy, L. Pál, P. Szabó, Phys. Chem. Solids 29, 101 (1968). 